International Journal of Modern Physics B

Vol. 17, No. 15 (2003) 2933-2935

(C) World Scientific Publishing Company

\title{
ERRATA
}

\section{STABILITY OF AN EXCITON BOUND TO AN IONIZED ACCEPTOR IN QUANTUM DOTS}

\author{
[Int. J. Mod. Phys. B. Vol. 17, No. 11 (2003), 2273-2279] \\ S. BASKOUTAS*, A. F. TERZIS and C. POLITIS \\ *bask@des.upatras.gr
}

Page 2273

(i) The abstract should be:-

Binding energy for an exciton (X) bound in a parabolic two-dimensional quantum dot by an acceptor impurity $A^{-}$located on the $z$-axis at a distance $d$ from the dot plane, are calculated by using the hartree formalism with a recently developed numerical method (PMM) for the solution of the Schrödinger equation. As our analysis indicates the binding energy decreases as the impurity distance from the dot plane decreases and furthermore no bound state exists for $d \rightarrow 0$. Finally, for fixed dot radius we have obtained a critical value of the mass ratio $\sigma^{c}(d)$ such that for $\sigma>\sigma^{c}(d)$ the system is unstable.

(ii) The final two sentences in this page should read:-

As a result it appear ${ }^{4}$ that the $\left(D^{+}, X\right)$ complex is stable if $\sigma \leq 0.454$ and that the $\left(A^{-}, X\right)$ is stable if $\sigma^{-1} \leq 0.454$. However, only few theoretical studies have been devoted to the $\left(D^{+}, X\right)$ and $\left(A^{-}, X\right)$ complexes in low-dimensional structure. ${ }^{5-9}$

Page 2279

(i) The last paragraph of Section 3 should not be there. (That paragraph is redundant)

\footnotetext{
* Corresponding author.
} 
(ii) The conclusions (Section 4) should be:-

In the present work we have studied the stability of $\left(A^{-}, X\right)$ complex in a two-dimensional GaAs QD. Using the Hartree formalism with PMM for the solution of the Schrödinger equation, we have obtained the binding energy $E_{b}$ of the complex. As our results indicate the binding energy decreases as the impurity distance from the dot plane decreases and furthermore no bound state exists for $d \rightarrow 0$. Finally, for a fixed dot radius $(R=7 \mathrm{~nm})$ we have obtained a critical value of the mass ratio $\sigma^{c}$ such that for $\sigma>\sigma^{c}$ the system is unstable.

We believe that these results, which are the continuation of the study on the stability of $\left(D^{+}, X\right)$ complex ${ }^{9}$ will be useful for the interpretation of future experiments on the stability of complexes in quantum dots.

Figure 1 (p. 2276) should be:

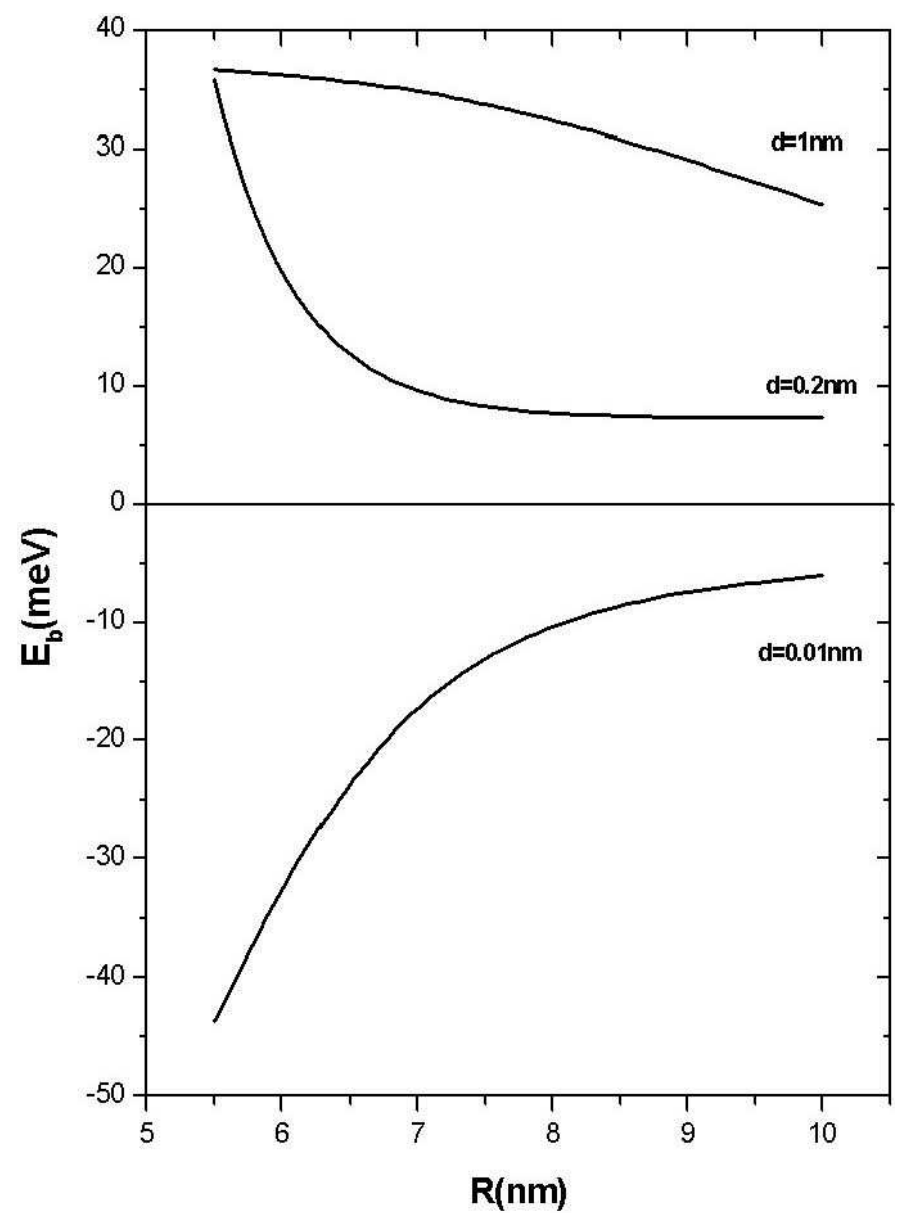


Figure 2 (p. 2277) should be:

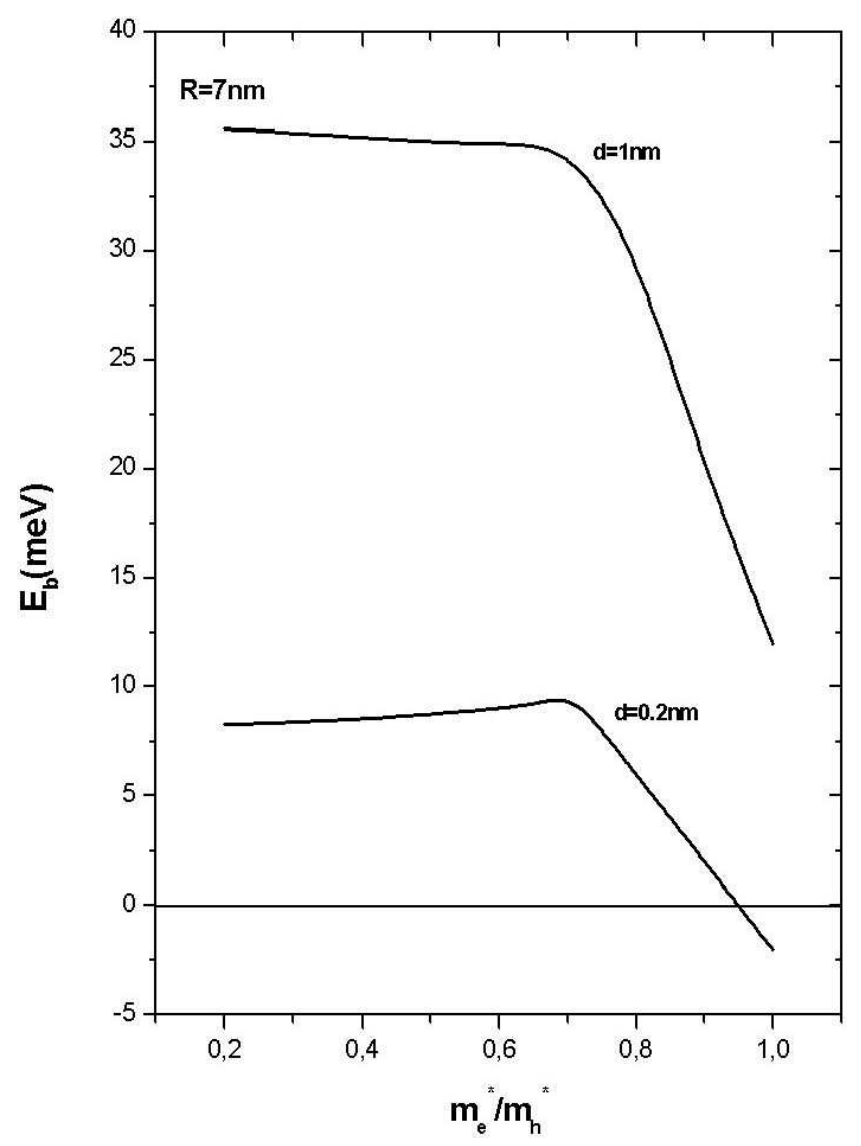

